\title{
Length effects of single-walled carbon nanotubes on pulmonary toxicity after intratracheal instillation in rats
}

\author{
Makoto Ema ${ }^{1,2}$, Hiroshi Takehara ${ }^{3}$, Masato Naya $^{1}$, Hiromichi Kataura ${ }^{4}$, Katsuhide Fujita ${ }^{1,2}$ \\ and Kazumasa Honda ${ }^{1,2}$
}

\author{
${ }^{1}$ Research Institute of Science for Safety and Sustainability, National Institute of Advanced Industrial Science and \\ Technology (AIST), 16-1 Onogawa, Tsukuba, Ibaraki 305-8569, Japan \\ ${ }_{2}^{2}$ Technology Research Association for Single Wall Carbon Nanotubes (TASC), \\ 16-1 Onogawa, Tsukuba, Ibaraki 305-8569, Japan \\ ${ }^{3}$ Public Interest Incorporated Foundation, BioSefety Research Center (BSRC), \\ 582-2 Shioshinden, Iwata, Shizuoka, 437-1213, Japan \\ ${ }^{4}$ Nanomaterials Research Institute, National Institute of Advanced Industrial Science and Technology (AIST), \\ Higashi 1-1-1, Tsukuba, Ibaraki 305-8565, Japan
}

(Received February 27, 2017; Accepted March 30, 2017)

\begin{abstract}
We aimed to evaluate the effects of the length of single-walled carbon nanotubes (SWCNTs) on pulmonary toxicity in rats. Each rat received a single intratracheal instillation of short (S-) (average length of $0.40 \mu \mathrm{m}$ ) or long (L-) (average length of $2.77 \mu \mathrm{m}$ ) SWCNTs at a dose of $1 \mathrm{mg} / \mathrm{kg}$ and was observed for the next 6 months. Neither S- nor L-SWCNTs affected clinical signs, body weight, or autopsy findings. An increase in lung weight was observed after instillation of S- or L-SWCNTs; however, lung weights were slightly higher in the rats that were administered the S-SWCNTs. Distinct differences in bronchoalveolar lavage fluid (BALF) composition were observed between the S- and L-SWCNTtreated rats as early as 7 days after the intratracheal instillations of the SWCNTs. The S-SWCNTs caused persistent lung injury and inflammation during the 6-month observational period. However, the L-SWCNTs induced minimal lung injury and inflammation. Although the S- and L-SWCNTs changed BALF parameters and histopathological features of the lung, the magnitudes of the changes observed after the S-SWCNT treatment were greater than the respective changes observed after the L-SWCNT treatment. These findings indicate that the severity of the pulmonary toxicity caused after intratracheal instillation of SWCNT depends on the length of the SWCNTs. It appears that shorter SWCNTs induce greater pulmonary toxicity than longer SWCNTs do.
\end{abstract}

Key words: Nanomaterial, Single-walled carbon nanotube, Lung toxicity/inflammation, Length effect, Intratracheal instillation

\section{INTRODUCTION}

Nanotechnology is a rapidly developing field in which new materials with size-dependent properties are created. As a result, there is a recent increase in exposure to nanomaterials (NMs). The distinctive and often unique properties of engineered NMs (ENMs) offer the promise of advances in a wide range of technologies. Because of their unique physico-chemical properties, such as high strength, reactivity, and conductivity, ENMs can be used in a wide range of products to improve performance and consumer benefits (Thomas, 2014). Human exposure to ENMs can occur at any stage during ENM synthesis/ manufacture, use, and disposal (Jiménez et al., 2014). The introduction of these novel materials into the work environment and consumer products necessitates safety evaluations on them as well as a clearer understanding of their potential impact on human health. Because cellular molecular machines and ENMs have comparable dimensions, one of the popular definitions of nanotoxicology puts an emphasis on the possibility of direct interaction and interference of ENMs with vital cellular processes (Shvedova et al., 2016). The potential of ENMs to interact with cellular molecular machines is a matter of

Correspondence: Makoto Ema (E-mail: ema-makoto@aist.go.jp,emamakoto@gmail.com) 
great concern.

Carbon nanotubes (CNTs) are an important new class of technological materials with excellent physico-chemical properties, which have received much attention lately. They are widely used in cutting-edge technologies and are one of the most commercially relevant classes of ENMs. CNTs potentially have the broadest range of applications, from composites to consumer electronics, energy storage, and health care among others (Singh and Song, 2012). CNTs include single-walled CNTs (SWCNTs), which are single sheets of graphite that are rolled to form seamless cylinders, and multi-walled CNTs (MWCNTs), which are composed of a number of sheets that are rolled up to form concentric tubes (Alexander, 2007). Presently, there is a knowledge gap between the increasing development and use of ENMs and CNTs, and their potential health risks.

It is well known that the physical and chemical properties, including size, shape, charge, agglomeration status, and metal impurity, of NMs affect their toxicity (Donaldson et al., 2006; Johnston et al., 2010). CNTs with high aspect ratios and long, thin, rigid, and biopersistent properties may induce pulmonary responses similar to those induced by asbestos (Poland et al., 2008; Braakhuis et al., 2014). Length is one of the critical factors underlying the potential toxicity of fibrous NMs (Ye et al., 1999; Hamilton et al., 2009). Therefore, fiber length is accepted as the major contributing factor in fiber pathogenicity (Schinwald et al., 2012). It has been reported that long fibers are cleared slowly from the lung, and therefore, are retained in the lung for long periods. This causes persistent lung burden, as the fibers are not easily engulfed by macrophages. This further leads to frustrated phagocytosis (Donaldson et al., 2006, 2010). In addition, curled and tangled CNTs, rather than straight fibers, probably do not induce pulmonary responses as asbestos does (Braakhuis et al., 2014). It has also been reported that intraperitoneal injection of long MWCNTs and long asbestos fibers cause inflammation in the peritoneal cavity in mice, whereas shorter and tangled MWCNTs and shorter asbestos fibers do not (Poland et al., 2008). However, the relationship between the length of SWCNTs and pulmonary toxicity remains unclear. In this study, we prepared short SWCNTs (S-SWCNTs) and long SWCNTs (L-SWCNTs), administered them to rats by intratracheal instillation, and evaluated the effects of their lengths on pulmonary toxicity in rats.

\section{MATERIALS AND METHODS}

\section{Preparation of test solutions}

Bulk Nikkiso SWCNTs (N-SWCNTs), which were synthesized by the catalytic chemical vapor deposition (CVD) method, were purchased from Nikkiso Co. Ltd. (Tokyo, Japan). The physico-chemical properties of bulk N-SWCNTs have been previously reported as follows: mean diameter (geometric mean), $1.8 \mathrm{~nm}$; BrunauerEmmett-Teller surface area, $878 \mathrm{~m}^{2} / \mathrm{g}$; and iron content, 43650 ppm (Ema et al., 2011; Morimoto et al., 2012). The bulk N-SWCNTs were dispersed in 10-fold diluted phosphate-buffered saline (PBS) containing 1\% salmonserum DNA (DNA; Wako Pure Chemical Industries Ltd., Osaka, Japan). The L-SWCNT solution was prepared using a homogenizer (Polytron PT 3100 D equipped with a $20-\mathrm{mm}$ blade; Kinematica, Luzen, Switzerland). To prevent shortening of the tube length, homogenization was started at a low speed $(8,000 \mathrm{rpm})$ and gradually increased to a high speed $(22,000 \mathrm{rpm})$ over $10 \mathrm{hr}$. The S-SWCNT solution was obtained from the L-SWCNT solution via ultrasonic homogenization (30\% output; Sniffier 250D; Branson, Danbury, CT, USA) of the latter for $5 \mathrm{hr}$, followed by purification by centrifugation to exclude small metal fragments detached from the tip of the homogenizer.

\section{Characterization of the SWCNTs}

Tube morphology was evaluated by transmission electron microscopy (JEM-1010; JEOL Ltd., Tokyo, Japan) at an acceleration voltage of $100 \mathrm{kV}$. Metal impurities in the SWCNT solutions were quantified by first subjecting the SWCNTs to microwave-assisted acid digestion, followed by the analysis of 28 elements using an inductively coupled plasma mass spectrometer (ICP-MS, 7500a; Agilent Technologies, Tokyo, Japan).

\section{Animals}

Specific pathogen-free male $\mathrm{Crl}: \mathrm{CD}(\mathrm{SD})$ rats (7-weekold) were purchased from Charles River Laboratories Japan, Inc. (Kanagawa, Japan). The rats were kept in an air-conditioned room (temperature, $22.7-23.3^{\circ} \mathrm{C}$; relative humidity, 38.6-67.8\%) under a 12/12 hr light/dark cycle and a ventilation rate of 12 air changes per hour. The animals were allowed free access to standard rodent pellets (CRF-1; Oriental Yeast Co., Tokyo, Japan) and tap water and were acclimated for 6 days before treatment.

The animal experiments were performed at the Public Interest Incorporated Foundation BioSafety Research Center (BSRC, Shizuoka, Japan) according to the Guidelines for Animal Experimentation (1987, Japan), the Law Concerning the Protection and Control of Animals (1973, Japan), and the Standards Relating to the Care and Management of Experimental Animals (1980, Japan). The study was approved by the Institutional Animal Care 
Pulmonary toxicity induced by single-walled carbon nanotubes

and Use Committee of BSRC and performed in accordance with the ethics criteria contained in the bylaws of the National Institute of Advanced Industrial Science and Technology (AIST).

\section{Experimental design}

At day 0 , each rat received a single intratracheal instillation of S-SWCNTs ( $1 \mathrm{mg} / \mathrm{mL} / \mathrm{kg})$, L-SWCNTs ( $1 \mathrm{mg} / \mathrm{mL} / \mathrm{kg}$ ), PBS (negative control, $1 \mathrm{~mL} / \mathrm{kg}$ ), $1 \%$ DNA in 10-fold diluted PBS (vehicle control, $1 \mathrm{~mL} / \mathrm{kg}$ ), or $5 \mu \mathrm{m}$ Min-U-Sil (Silica) (positive control; $5 \mathrm{mg} / \mathrm{mL} / \mathrm{kg}$; U.S. Silica, Frederick, MD, USA). Inhaled crystalline silica is a known carcinogen in humans and animals. It has been reported that crystalline silica causes adenocarcinomas and squamous cell carcinomas after its inhalation or intratracheal instillation in rats (WHO, 2000). In the present study, intratracheal instillation was performed using a microsprayer (Model IA-1B; Penn-Century Inc., Wyndmoor, PA, USA) under anesthesia, which was induced by the inhalation of $5 \%$ isoflurane. Following the intratracheal instillation, the general condition and viability of the rats were observed once a day until autopsy was performed. The body weight of each rat was measured before the instillation (day 0 ) and once a week post instillation up to 6 months. The left lung was weighed and the left lung, liver, left kidney, spleen, and brain were histopathologically examined. Five rats per group were examined at each time point.

\section{Analysis of bronchoalveolar lavage fluid (BALF)}

BALF analysis was conducted as previously described (Fujita et al., 2015a). Briefly, the rats were euthanized by isoflurane anesthesia and their lungs were removed. BALF was obtained from the right lung as follows. First, the left bronchus was ligated with sutures and the right bronchus was cannulated. Next, $3 \mathrm{~mL}$ of physiological saline was introduced into the lungs, which were then gently manipulated, followed by the withdrawal of lavage fluid. This procedure was repeated 3 times. The recovered BALF was centrifuged and the biochemical composition of the supernatant was analyzed as follows. The levels of total protein and lactate dehydrogenase (LDH) were measured using an automatic analyzer (7170; Hitachi High-Technologies Corporation, Tokyo Japan). The levels of granulocyte macrophage colony-stimulating factor, interferon- $\gamma$, tumor necrosis factor (TNF)- $\alpha$, macrophage chemoattractant protein (MCP)-1, growth-related oncogene/keratinocyte chemoattractant (GRO-KC), and interleukin (IL)-1 $\alpha, 1 \beta, 2,4,5,6,10,12$ (p70), and 18 were measured using a MILLIPLEX MAP Rat Cytokine/ Chemokine Panel and a Luminex 200 System (Merck
Millipore, Billerica, MA, USA). The cell fraction was suspended in PBS and the total cell count was determined using a hematology system (ADVIA 120; Siemens Healthcare Diagnostics, Inc., Tokyo, Japan). The numbers of neutrophils, macrophages, lymphocytes, and eosinophils were determined by May-Grünwald-Giemsa staining.

\section{Histopathological examination}

The left lung, liver, left kidney, spleen, and brain were fixed in $10 \%$ neutral buffered formalin. All the fixed tissues were routinely processed, embedded in paraffin, sectioned into $3-\mu \mathrm{m}$ pieces, and stained with hematoxylin and eosin for histopathological examination.

\section{Statistical analysis}

Pairwise comparisons were performed using the Tukey-Kramer test (Tukey, 1953; Kramer, 1956; Hayter, 1984) with the Shaffer-Royen procedure (Shaffer, 1986; Royen, 1989). A probability level of $p<0.05$ was used as the criterion of significance.

\section{RESULTS}

\section{Characterization of the SWCNTs}

In the test solutions, SWCNTs were presented in bundled form with each bundle consisting of several single fibers. The length distributions of the S- and L-SWCNTs are shown in Fig. 1. The lengths of the SWCNTs were measured from digital images acquired using an atomic force microscope (S-image; SII, Chiba, Japan) equipped with the UTHSCSA ImageTool software (UT Health Science Center San Antonio, TX, USA). The average lengths and standard deviations of the S-SWCNTs and L-SWCNTs were $0.40 \pm 0.29 \mu \mathrm{m}(\mathrm{n}=443$ from 4 images) and $2.77 \pm 3.22 \mu \mathrm{m}(\mathrm{n}=477$ from 6 images $)$, respectively.

The amounts of Fe, Co, Ni, and V impurities were 45, $12,0.16$, and $0.55 \mu \mathrm{g} / \mathrm{g}$, respectively, in the S-SWCNT solution and $62,24,0.28$, and $0.011 \mu \mathrm{g} / \mathrm{g}$, respectively, in the L-SWCNT solution.

\section{General condition and the body and lung weights of the rats}

During the 6-month observational period, no treatment-related clinical signs of toxicity were observed in any of the rats. In addition, the intratracheal instillations of S-SWCNTs, L-SWCNTs, and Silica did not result in any changes in body weight. The relative weights of the left lungs of the rats are presented in Fig. 2. Compared to the negative control, the relative weights of the left lung 
S-SWCNTS

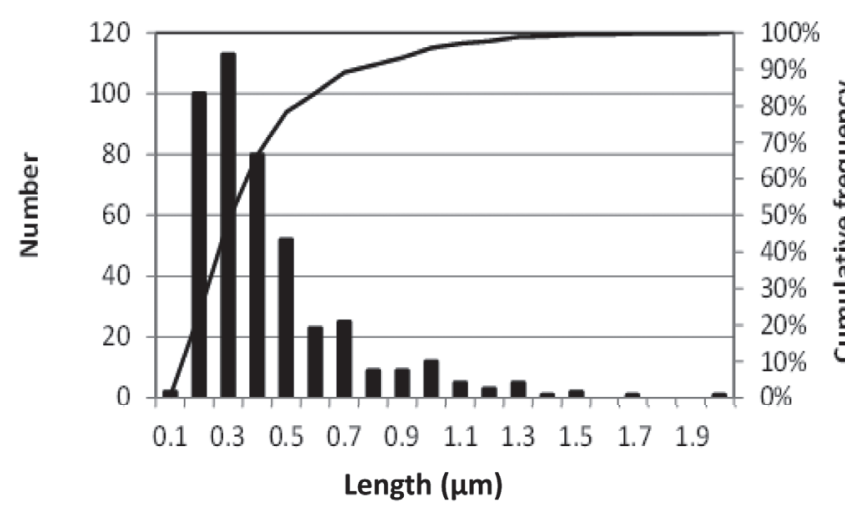

L-SWCNTS

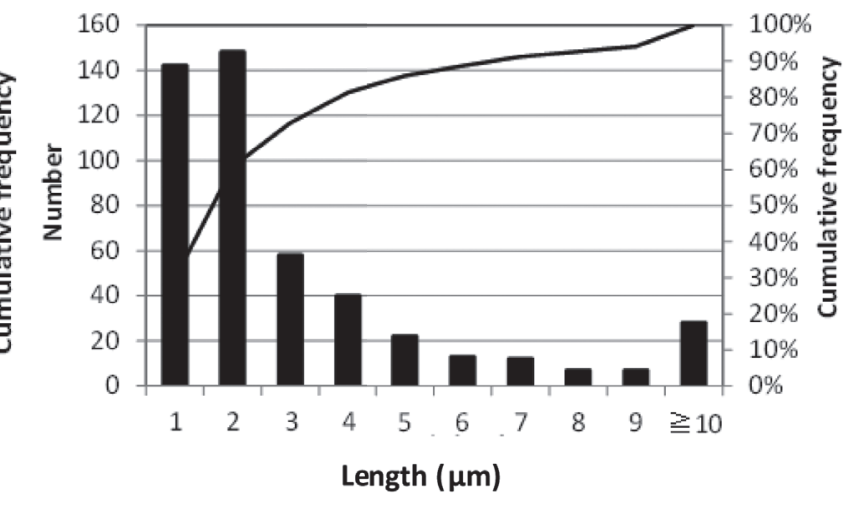

Fig. 1. Length distributions of S-SWCNTs and L-SWCNTs. The lengths of the nanotubes were measured from digital images acquired from an atomic force microscope.

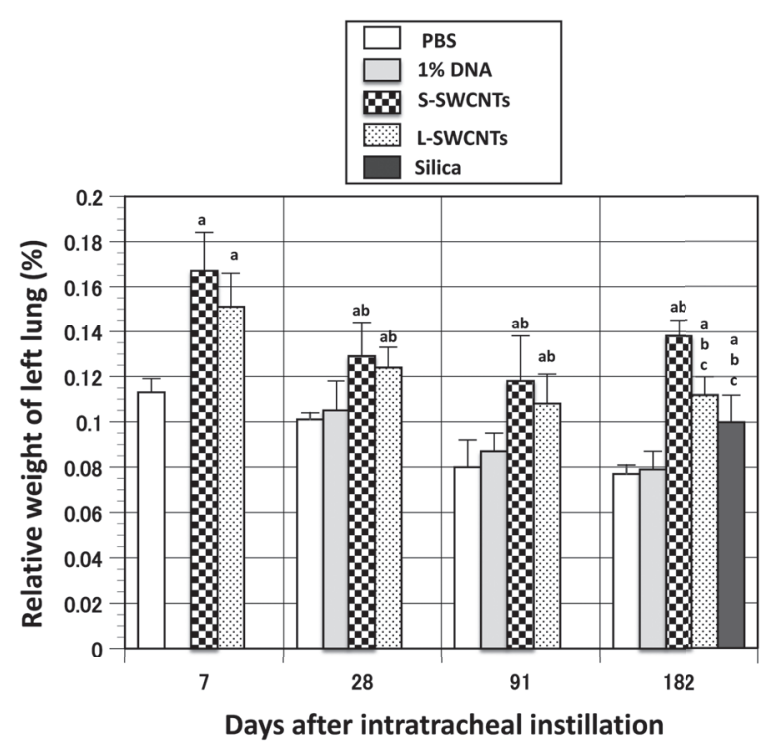

Fig. 2. Relative weights of the left lungs (g/100 g of body weight) of rats after receiving a single intratracheal instillation of SWCNTs. Values are presented as mean \pm standard deviation (S.D.). a, b, and c denote statistically significant differences $(p<0.05)$ when compared with the negative control (PBS), vehicle control (1\% DNA), and S-SWCNT-treated groups, respectively.

were significantly increased after the S- and L-SWCNT treatments at days 7, 28, 91, and 182 and after the Silica treatment at day 182 . Compared to the vehicle control group, the relative lung weights were heavier in the S- and L-SWCNT-treated groups at days 28, 91, and 182, and in the Silica-treated group at day 182. At day 182, the relative lung weights in the L-SWCNT- and Silica-treated groups were lower than those in the S-SWCNT-treated group.

\section{Gross findings}

No abnormalities were observed in the negative and vehicle control groups at any time point. Black or brown spots were found in the lungs of all the S- and L-SWCNT-treated rats at all time points. However, brown spots or nodules were observed in the lungs of all the Silicatreated rats at day 182 .

\section{BALF analysis}

The total number of nucleated cells and ratio of neutrophils in each BALF sample are shown in Fig. 3. Compared to the negative control group, the total number of nucleated cells was significantly higher in the S-SWCNT-treated group (at days 7, 28, 91, and 182) and the Silica-treated group (at day 182). Compared to the vehicle control group, the total number of nucleated cells was significantly higher in the S-SWCNT-treated group (at days 28, 91, and 182) and the Silica-treated group (at day 182). Furthermore, at days 91 and 182, the total numbers of nucleated cells in the L-SWCNT-treated group were lower than the respective values in the S-SWCNT-treated group.

A significantly higher ratio of neutrophils was found in the S-SWCNT-treated group at days 7, 28, 91, and 182, as well as in the Silica-treated group at day 182, when compared to the respective ratios in the negative control group. Compared to the vehicle control group, higher neutrophil ratios were observed in the S-SWCNT-treated groups at days 28, 91, and 182 and in the Silica-treated 
Pulmonary toxicity induced by single-walled carbon nanotubes

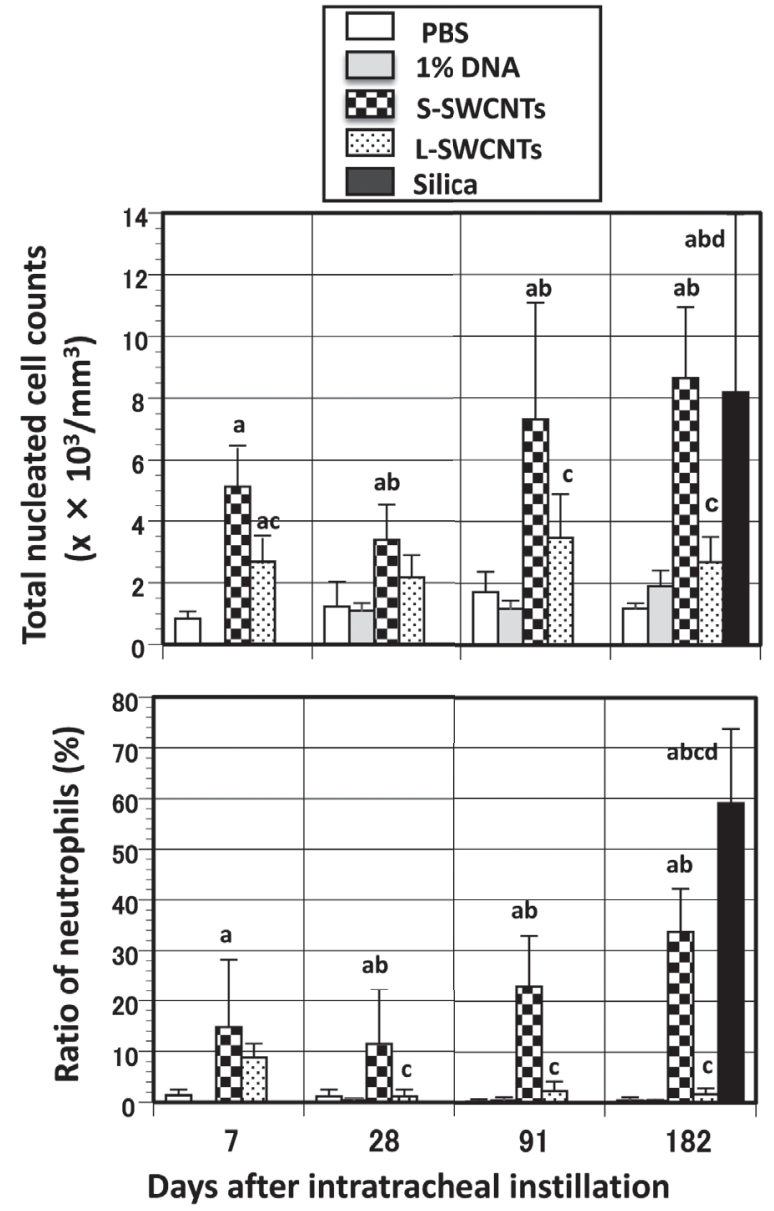

Fig. 3. Total nucleated cell counts and ratio of neutrophils in BALF samples after a single intratracheal instillation of SWCNTs in rats. Values are presented as mean \pm S.D. a, b, c, and d denote statistically significant differences $(p<0.05)$ when compared with the negative control (PBS), vehicle control (1\% DNA), S-SWCNTtreated, and L-SWCNT-treated groups, respectively.

group at day 182. Moreover, at days 28, 91, and 182, the neutrophil ratios in the L-SWCNT-treated group were lower than the respective ratios in the S-SWCNT-treated group.

The total protein and LDH levels in the BALF samples are presented in Fig. 4. Compared to the negative control treated group, the total protein levels were significantly higher at days 91 and 182 in the S- and L-SWCNT-treated groups and at day 182 in the Silica-treated group. However, at day 91, the total protein levels in the L-SWCNTtreated group were significantly lower than those in the S-SWCNT-treated group.

Furthermore, compared to the negative control group,

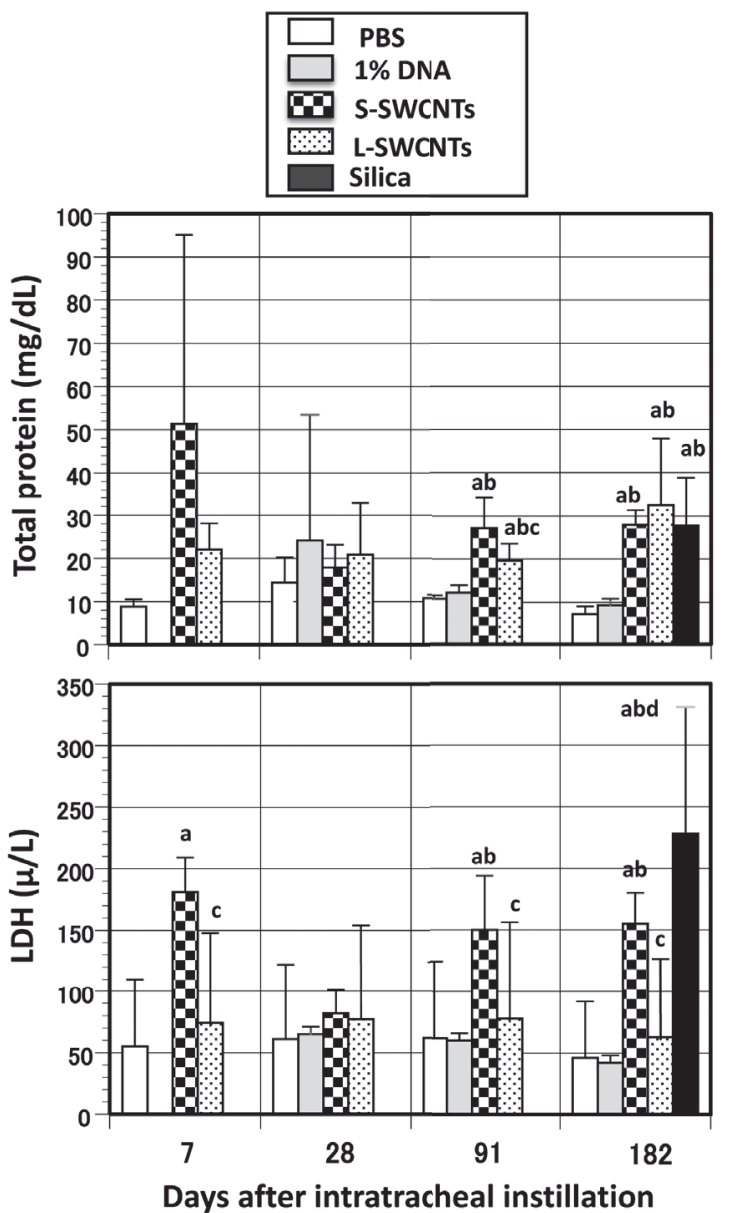

Fig. 4. Total protein and LDH levels in BALF samples after a single intratracheal instillation of SWCNTs in rats. Values are presented as mean \pm S.D. $a, b$, c, and $d$ denote statistically significant differences $(p<0.05)$ when compared with the negative control (PBS), vehicle control (1\% DNA), S-SWCNT-treated, and LSWCNT-treated groups, respectively.

the LDH levels were significantly higher at days 7, 91, and 182 in the S-SWCNT-treated group and at day 182 in the Silica-treated group. Compared to the vehicle control group, significantly higher LDH levels were observed in the S-SWCNT-treated group at days 91 and 182 and in the Silica-treated group at day 182. Additionally, the LDH levels in the L-SWCNT-treated group at day 91 and 182 were significantly lower than those at the respective days in the S-SWCNT-treated group.

Table 1 shows the levels of MCP-1, IL-18, and GRO$\mathrm{KC}$ in the BALF samples. At day 91, the MCP-1 level in the S-SWCNT-treated group was significantly higher than that in the vehicle control group. Furthermore, at day 91 , 
M. Ema et al.

Table 1. The levels of MCP-1, IL-18, and GRO/KC in BALF after a single intratracheal instillation of SWCNTs in rats.

\begin{tabular}{|c|c|c|c|c|c|}
\hline $\begin{array}{l}\text { Day of } \\
\text { autopsy }\end{array}$ & Treatment & No. of rats & $\mathrm{MCP}-1(\mathrm{pg} / \mathrm{mL})$ & IL-18 (pg/mL) & GRO-KC (pg/mL) \\
\hline \multirow[t]{5}{*}{ atiop oy } & PBS (negative control) & 5 & $<1.2$ & $138.2 \pm 42.6$ & $237.9 \pm 45.8$ \\
\hline & $1 \%$ DNA (vehicle control) & 0 & & & \\
\hline & S-SWCNTs & 5 & $173.7 \pm 150.6$ & $200.9 \pm 46.6$ & $1829.0 \pm 714.2^{\mathrm{a}}$ \\
\hline & L-SWCNTs & 5 & $24.9 \pm 11.7$ & $149.6 \pm 25.7$ & $376.1 \pm 124.5^{\mathrm{c}}$ \\
\hline & Silica & 0 & & & \\
\hline \multirow{5}{*}{28} & PBS (negative control) & 5 & $6.4(1)$ & $96.9 \pm 24.0$ & $169.8 \pm 30.9$ \\
\hline & $1 \%$ DNA (vehicle control) & 5 & $12.5 \pm 0.8(3)$ & $128.1 \pm 26.3$ & $214.7 \pm 47.7$ \\
\hline & S-SWCNTs & 5 & $15.4 \pm 4.4(3)$ & $111.2 \pm 50.4$ & $223.6 \pm 64.2$ \\
\hline & L-SWCNTs & 5 & $17.1(2)$ & $142.1 \pm 74.0$ & $162.6 \pm 42.0$ \\
\hline & Silica & 0 & & & \\
\hline \multirow{5}{*}{91} & PBS (negative control) & 5 & $8.2(1)$ & $156.0 \pm 35.1$ & $248.5 \pm 127.3$ \\
\hline & $1 \%$ DNA (vehicle control) & 5 & $19.1(2)$ & $159.7 \pm 52.6$ & $330.0 \pm 193.3$ \\
\hline & S-SWCNTs & 5 & $140.2 \pm 65.0^{\mathrm{b}}$ & $336.0 \pm 154.7^{\mathrm{a}, \mathrm{b}}$ & $2262.5 \pm 1110.2^{\mathrm{a}, \mathrm{b}}$ \\
\hline & L-SWCNTs & 5 & $26.9 \pm 8.0(4)^{\mathrm{c}}$ & $188.1 \pm 70.3^{c}$ & $256.3 \pm 84.4^{c}$ \\
\hline & Silica & 0 & & & \\
\hline \multirow{5}{*}{182} & PBS (negative control) & 5 & $4.7 \pm 0.8(3)$ & $136.5 \pm 38.7$ & $252.8 \pm 104.1$ \\
\hline & $1 \%$ DNA (vehicle control) & 5 & $3.5(2)$ & $116.3 \pm 28.4$ & $294.9 \pm 95.1$ \\
\hline & S-SWCNTs & 5 & $406.5 \pm 71.0$ & $523.0 \pm 74.6$ & $2470.7 \pm 992.9^{\mathrm{a}, \mathrm{b}}$ \\
\hline & L-SWCNTs & 5 & $16.3 \pm 9.5$ & $199.7 \pm 69.3$ & $328.8 \pm 217.9^{c}$ \\
\hline & Silica & 5 & $820.9 \pm 516.5^{\mathrm{a}, \mathrm{b}, \mathrm{d}}$ & $968.9 \pm 510.3^{\mathrm{a}, \mathrm{b}, \mathrm{c}, \mathrm{d}}$ & $1119.6 \pm 653.1^{\mathrm{c}}$ \\
\hline
\end{tabular}

Values are presented as mean \pm S.D. The numbers in parentheses represent the number of rats examined. a, b, c, and d denote statistically significant differences $(p<0.05)$ when compared with the negative control (PBS), vehicle control $(1 \%$ DNA), S-SWCNT-treated, and L-SWCNT-treated groups, respectively.

the MCP-1 levels in the L-SWCNT-treated group were significantly lower than that in the S-SWCNT-treated group. Additionally, at day 182, the MCP-1 levels in the Silica-treated group were significantly higher than those in the negative control, vehicle control, and L-SWCNTtreated groups.

At day 91, the levels of IL-18 were significantly higher in the S-SWCNT-treated group than those in the negative and vehicle control groups. However, at day 91, the IL-18 levels in the L-SWCNT-treated group were significantly lower than those in the S-SWCNT-treated group. At day 182, the IL-18 levels in the Silica-treated group were significantly higher than those in the negative control, vehicle control, and S- and L-SWCNT-treated groups.

Furthermore, the levels of GRO-KC in the S-SWCNTtreated group were significantly higher at days 7, 91, and 182 than those at the respective days in the negative control group. Similarly, GRO-KC levels at days 91 and 182 in the S-SWCNT-treated group were significantly higher than those at the respective days in the vehicle control group. Additionally, GRO-KC levels in the L-SWCNTtreated group were significantly lower than those in the S-SWCNT-treated group at days 91 and 182. At day 182, GRO-KC levels in the Silica-treated group were signifi- cantly lower than those in the S-SWCNT-treated group.

It was however observed that the levels of the other cytokines in BALF remained unchanged after the S-SWCNT, L-SWCNT, and Silica treatments.

\section{Histopathological findings}

No histopathological changes were observed in the brain, spleen, liver, or kidney in any group. Histopathological changes in the lung that were caused by exposure to the S-SWCNTs or L-SWCNTs are shown in Fig. 5. Table 2 summarizes the histopathological findings and their severity scores at each time point after the SWCNT treatments. In the PBS-treated and vehicle control groups, a slight accumulation of lipid-laden macrophages in the alveoli, slight and transient hemorrhage, and/ or perivascular eosinophilic infiltration were observed. Intratracheal instillation of S-SWCNTs in the rats caused hemorrhage and the accumulation of macrophages containing lipids and the test substance in the alveoli at days 7-182. There was also perivascular infiltration of eosinophils at days 7-91, whereas granulomas, alveolar proteinosis, alveolar infiltration of neutrophils, and bronchioloalveolar proliferation were observed at days 91 and 182. At days 7-182, the L-SWCNT treatment induced 


\section{Day 7 after instillation}
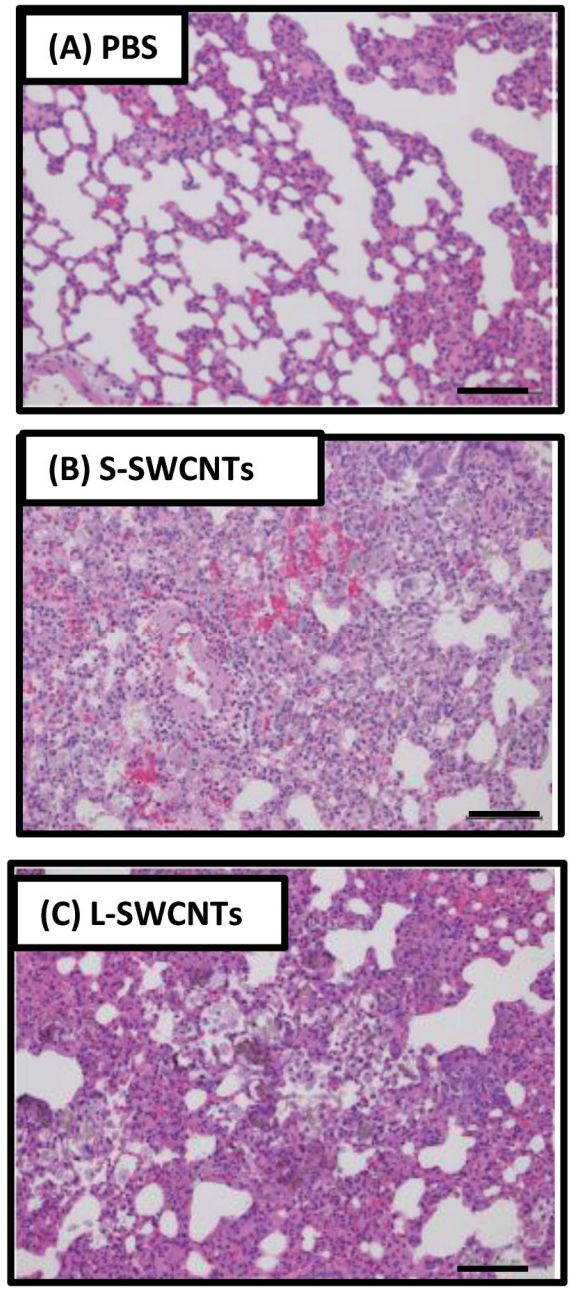

Day 182 after instillation
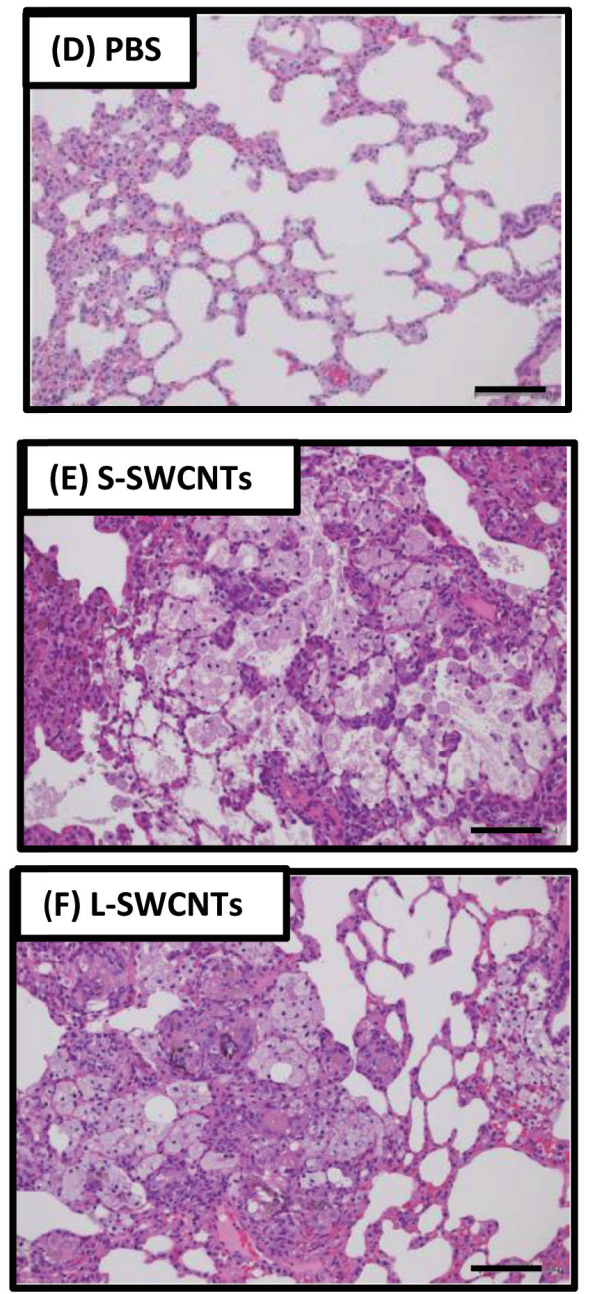

Fig. 5. Histopathology of the rat lung (HE staining; scale bar, $100 \mu \mathrm{m}$ ). (A) Seven days after a single intratracheal instillation of PBS (negative control). No significant lesions found. (B) Seven days after a single intratracheal instillation of S-SWCNTs. Hemorrhage, perivascular infiltration of eosinophils, and accumulation of macrophages containing lipids and the test substance in the alveoli were observed. (C) Seven days after a single intratracheal instillation of L-SWCNTs. Hemorrhage, granulomas, and accumulation of macrophages containing lipids and the test substance in the alveoli were observed. (D) Six months after a single intratracheal instillation of PBS. No significant lesions were observed. (E) Six months after a single intratracheal instillation of S-SWCNTs. Granulomas and a marked accumulation of macrophages containing lipids and the test substance in the alveoli were observed. (F) Six months after a single intratracheal instillation of L-SWCNTs. Granulomas containing the test substance were observed.

hemorrhage, accumulation of lipid-laden macrophages in the alveoli, and granulomas; however, it induced the accumulation of macrophages containing the test substance in the alveoli at days 7-91, perivascular infiltration of eosinophils at day 7 , and mineralization in granulomas at days 28-182.

\section{DISCUSSION}

In this study, we determined the pulmonary toxicities of S- and L-SWCNTs after single intratracheal instillations in rats. Especially, we evaluated the effect of SWCNT length on pulmonary toxicity. To date, majority of animal studies on SWCNTs have been performed via airway exposure, because this exposure route is the most impor- 
Table 2. Severity scores determined from the results of the pulmonary histopathology after a single intratracheal instillation of SWCNTs in rats.

\begin{tabular}{|c|c|c|c|c|c|c|}
\hline Findings & $\begin{array}{l}\text { Days after } \\
\text { intratracheal } \\
\text { instillation }\end{array}$ & PBS & $1 \%$ DNA & S-SWCNTs & L-SWCNTs & Silica \\
\hline \multirow{4}{*}{ Hemorrhage } & 7 & 0 & & 0.8 & 0.4 & \\
\hline & 28 & 0 & 0.2 & 0.6 & 0.6 & \\
\hline & 91 & 0 & 0 & 0.2 & 0.8 & \\
\hline & 182 & 0 & 0 & 0.2 & 1.2 & 0.4 \\
\hline Accumulation of lipid-laden & 7 & 0.2 & & 0.2 & 1.8 & \\
\hline \multirow{3}{*}{ macrophages in the alveoli } & 28 & 0.2 & 0.6 & 2.0 & 2.2 & \\
\hline & 91 & 0.2 & 0.4 & 2.8 & 3.0 & \\
\hline & 182 & 0.4 & 0.2 & 3.0 & 2.6 & 3.0 \\
\hline Accumulation of macrophages & 7 & 0 & & 1.6 & 1.6 & \\
\hline \multirow[t]{3}{*}{ engulfing test substances in the alveoli } & 28 & 0 & 0 & 2.4 & 1.2 & \\
\hline & 91 & 0 & 0 & 2.2 & 0.4 & \\
\hline & 182 & 0 & 0 & 1.6 & 0 & 0 \\
\hline \multirow[t]{4}{*}{ Granuloma } & 7 & 0 & & 0 & 0.4 & \\
\hline & 28 & 0 & 0 & 0 & 0.6 & \\
\hline & 91 & 0 & 0 & 0.2 & 1.6 & \\
\hline & 182 & 0 & 0 & 0.8 & 1.8 & 0 \\
\hline \multirow{4}{*}{ Mineralization } & 7 & 0 & & 0 & 0 & \\
\hline & 28 & 0 & 0 & 0 & 0.4 & \\
\hline & 91 & 0 & 0 & 0 & 0.4 & \\
\hline & 182 & 0 & 0 & 0 & 0.8 & 0 \\
\hline \multirow[t]{4}{*}{ Perivascular infiltration of eosinophils } & 7 & 0 & & 1.6 & 0.4 & \\
\hline & 28 & 0.4 & 0.2 & 0.2 & 0 & \\
\hline & 91 & 0 & 0 & 0.2 & 0 & \\
\hline & 182 & 0 & 0 & 0 & 0 & 0 \\
\hline \multirow[t]{4}{*}{ Alveolar proteinosis } & 7 & 0 & & 0 & 0 & \\
\hline & 28 & 0 & 0 & 0 & 0 & \\
\hline & 91 & 0 & 0 & 0.2 & 0 & \\
\hline & 182 & 0 & 0 & 1.4 & 0 & 3.0 \\
\hline \multirow[t]{4}{*}{ Infiltration of neutrophils in the alveoli } & 7 & 0 & & 0 & 0 & \\
\hline & 28 & 0 & 0 & 0 & 0 & \\
\hline & 91 & 0 & 0 & 0.6 & 0 & \\
\hline & 182 & 0 & 0 & 1.0 & 0 & 1.6 \\
\hline \multirow[t]{4}{*}{ Bronchioloalveolar proliferation } & 7 & 0 & & 0 & 0 & \\
\hline & 28 & 0 & 0 & 0 & 0 & \\
\hline & 91 & 0 & 0 & 0.2 & 0 & \\
\hline & 182 & 0 & 0 & 1.0 & 0 & 2.4 \\
\hline
\end{tabular}

Severity scores: 0 , not remarkable; 1 , slight; 2 , moderate; and 3, marked. The scores were determined for each animal and were based on findings from relative evaluations of the pulmonary lesions. The average severity score was then calculated for each rat group $(\mathrm{n}=5)$.

tant for CNT entry into the body and the key exposure route in fiber pathogenicity. Inhalation is the gold standard for determining the potential toxicity of an inhalant and represents the main route of exposure to NMs in an occupational setting. Intratracheal instillation is recognized as a drug administration procedure that usually reproduces the effects of inhalation well and can provide relevant information regarding the basic toxicity of an inhal- able material (Driscoll et al., 2000; Costa et al., 2006). It has been reported that the outcomes of inhalational exposure to SWCNTs, which includes pulmonary toxicity, are similar to those observed after a bolus administration (pharyngeal aspiration or intratracheal instillation) of SWCNTs (Shvedova et al., 2008, 2009; Castranova and Mercer, 2012).

Landsiedel et al. (2014) noted that BALF neutrophil 
Pulmonary toxicity induced by single-walled carbon nanotubes

counts, total protein, and histopathological findings are important parameters for evaluating the pulmonary toxicities of inhaled NMs. We also demonstrated in our previous study that counting and differentiating among cells, determining the levels of cytokines in BALF, and histopathological examination of the lungs are very important parameters in assessing pulmonary toxicity after exposure to SWCNTs via the airway (Ema et al., 2016). These endpoints were measured in the present study.

Distinct differences in BALF composition were observed between the S- and L-SWCNT-treated rats as early as 7 days after the intratracheal instillations of the SWCNTs. The S-SWCNTs caused persistent lung injury, which was evidenced by continuously elevated levels of LDH and total protein in the BALF, and inflammation, which was evidenced by continuously elevated levels of MCP-1, IL-18, and GRO-KC in BALF, during the 6-month observational period. However, the L-SWCNTs induced lung injury and inflammation only minimally. The amount of total protein in BALF increased during the late phase of the observational period after the L-SWCNT treatment. In addition, the levels of inflammatory markers slightly increased and histopathological changes were observed. Overall, the magnitudes of the changes observed after the S-SWCNT treatment were greater than the respective changes observed after the L-SWCNT treatment. These findings suggest that, after intratracheal instillation, S-SWCNTs cause acute and persistent pulmonary injury and inflammation, whereas L-SWCNTs induce weak inflammatory responses. The most important finding of the present study is that the severity of pulmonary toxicity caused after airway exposure to an SWCNT depends on the length of the SWCNT. Specifically, shorter SWCNTs cause higher pulmonary toxicities than longer SWCNTs do.

In our previous study, super-growth SWCNTs (SGSWCNTs) were obtained from the Technology Research Association for SWCNT (Japan) and evaluated for their size effects on pulmonary toxicity (Fujita et al., 2015a). SWCNTs that were in the form of relatively thin, short, and linear bundles (S-SG-SWCNTs, $0.51 \mu \mathrm{m}$ in average length) or relatively thick, long, and linear bundles (L-SGSWCNTs, $1.67 \mu \mathrm{m}$ in average length) were administered to rats by a single intratracheal instillation. The rats were then observed until 3 months after the instillations. The S-SG-SWCNTs induced higher inflammatory responses in the lungs than the L-SG-SWCNTs did. Furthermore, after the S-SG-SWCNT instillation, changes in the expression levels of genes associated with inflammation, cell proliferation, and immune system functions were evaluated throughout the observational period. On the other hand, after the L-SG-SWCNT treatment, changes in the expression levels of many genes were observed at day 1; however, this differential regulation was decreased by day 3 . The authors suggested that S-SG-SWCNTs induce pulmonary inflammation with a slow recovery, whereas L-SGSWCNTs induce acute inflammation and cellular responses in alveolar macrophages.

Furthermore, the pulmonary effects of short N-SWCNTs $(0.69 \mu \mathrm{m}$ in average length) have been evaluated in rats after intratracheal instillation. The N-SWCNTs, whether singly instilled (at 0.2 or $1.0 \mathrm{mg} / \mathrm{kg}$ ) or repeatedly instilled (once a week for 5 weeks at 0.04 or $0.2 \mathrm{mg} / \mathrm{kg}$ ), induced acute pulmonary inflammation. However, no DNA damage in lung tissues was observed in comet assays at any of the doses studied (Naya et al., 2012). In another study, following a single intratracheal instillation of N-SWCNTs at 0.66 or $1.32 \mathrm{mg} / \mathrm{kg}$, persistent ( 6 months) pulmonary inflammation was observed at both doses. At the $1.32-\mathrm{mg} / \mathrm{kg}$ dose, pulmonary inflammation became severe and a mild fibrotic change was observed at 6 months (Morimoto et al., 2012). Furthermore, it has been reported that at day 754 after a single intratracheal instillation of N-SWCNTs at 0.2 or $0.4 \mathrm{mg} / \mathrm{rat}$, the N-SWCNTs were retained in the alveoli and bronchioles of the animals (Fujita et al., 2015b). The authors suggested that intratracheal instillation of short N-SWCNTs could induce acute and persistent pulmonary inflammation. The results of the present study support the findings of the above-mentioned previous studies since the S-SWCNTs induced pulmonary inflammation that persisted throughout the 6-month observational period.

The histopathological examination of the lungs revealed that granulomas were developed at days 91 and 182 after the S-SWCNT treatment and at days 7-182 after the L-SWCNT treatment. Moreover, Warheit et al. (2004) observed that intratracheal instillation of SWCNTs resulted in granuloma formation in the lungs of rats. However, pulmonary biomarkers of inflammation, cell proliferation, and cytotoxicity were not observed during the 3-month observational period after the instillation. These observations are quite similar to ours in the present study. SWCNT agglomerates induce granuloma formation, which leads to morphological/structural isolation of SWCNT agglomerates within the lung tissues. This presumably makes the SWCNTs less damaging to the surrounding pulmonary tissues. In addition, once agglomerated SWCNTs in lung tissues are walled off by cuboidal cells, they are less likely to induce inflammatory responses (Murray et al., 2012). These findings suggest that rapid isolation of L-SWCNTs from surrounding pulmonary tissues may weaken the inflammatory responses evoked by 
the L-SWCNTs.

Metal impurities can play a role in the pulmonary toxicity caused by NMs. As a result, the toxicity of CNTs is closely associated with iron impurities, because iron can generate reactive oxygen species (ROS) (Murray et al., 2009; Ge et al., 2012). A more severe pulmonary toxicity has been reported to be caused by SWCNTs with high metal content (Ge et al., 2012). Shvedova et al. (2014a) showed that pretreatment of SWCNTs with a metal chelator attenuated the pulmonary toxicity of SWCNTs. In addition, the authors suggested that the generation of free radicals is involved in SWCNT-induced oxidative stress/ inflammation in the lungs and that metals have an important role in this process. On the other hand, Lam et al. (2004) noted that iron content did not influence the relative lung toxicity of SWCNTs. An in vitro study showed that impurity-free CNTs induced a high level of ROS production and suggested that residual metals from SWCNT manufacture are not a definite parameter for intracellular ROS generation (Fujita et al., 2013). In the present study, S-SWCNTs induced a more severe pulmonary toxicity despite having a slightly lower iron content than the L-SWCNTs did. In our previous studies, the pulmonary toxicity of short-tube N-SWCNTs, which contained a high amount iron as an impurity (13700 ppm), was studied in rats (Naya et al., 2012; Fujita et al., 2015b). We observed that the pulmonary inflammation induced by N-SWCNTs in the previous studies is not markedly different from that induced by S-SWCNTs in the present study. These findings suggest that the iron content in a SWCNT formulation may not be involved in the induction of inflammatory responses by SWCNTs. At present, however, it is difficult to draw any firm conclusions regarding the role of metal impurities in the pulmonary toxicity of SWCNTs. Further studies, therefore, are needed to clarify the role of metal impurities in induction of inflammatory responses by SWCNTs.

Although many studies have been performed on the effects of airway exposure to SWCNTs on pulmonary toxicity, only a few were long-term observational studies. In a one-year observational study on purified HiPco SWCNTs (0.1-1 $\mu \mathrm{m}$ in length) in mice, no increased incidence of lung tumor was reported after administration of the SWCNTs by inhalation or pharyngeal aspiration (Shvedova et al., 2014b). Furthermore, in a study conducted in rats, no pulmonary tumor was detected 754 days after intratracheal instillation of SWCNTs (average length, $0.69 \mu \mathrm{m}$ ) (Fujita et al., 2015b). However, a substantial number of SWCNTs as well as inflammation and fibrotic changes were observed in the lungs of the rats at 1-year post exposure to the SWCNTs
(Shvedova et al., 2014b; Fujita et al., 2015b). In the present study, we showed that S-SWCNTs induced persistent pulmonary inflammation and L-SWCNTs caused increased levels of total protein in BALF during the later phase of the 6-month observational period. Furthermore, chronic inflammation and fibrotic changes in the lungs are considered as significant risk factors for pulmonary carcinogenesis (Hubbard et al., 2000; Shvedova et al., 2008). However, to our knowledge, no study has yet shown the potential carcinogenicity of SWCNTs. We are therefore currently conducting a 2-year observational study to evaluate the lung carcinogenicity of SWCNTs after intratracheal instillations of S- and L-SWCNTs in rats.

In conclusion, our 6-month observational study in rats revealed that $\mathrm{S}-\mathrm{SWCNT}$ are more toxic to the lungs than are L-SWCNTs. After intratracheal instillations, the S-SWCNTs induced persistent inflammation and toxicity in the lungs of the animals, whereas the L-SWCNTs induced a slight inflammation.

\section{ACKNOWLEDGMENTS}

The authors would like to thank Dr. Junko Nakanishi for her advice on the study design, Dr. Shuji Abe for his support throughout the project, and Dr. Kazuhiro Yamamoto for his helpful discussions. This study was based on the results obtained from a project, entitled "Innovative Carbon Nanotubes Composite Materials Project toward Achieving a Low-Carbon Society (P10024)," which was commissioned by the New Energy and Industrial Technology Development Organization (NEDO) in Japan.

Conflict of interest---- The authors declare that there is no conflict of interest.

\section{REFERENCES}

Alexander, A.J. (2007): Carbon nanotube structures and compositions: implications for toxicological studies. In: MonteiroRiviere, N.A., Tran, C.L., (Eds.), Nanotoxicology: Characterization, Dosing and Health Effects. Informa Healthcare, New York, pp.7-18.

Braakhuis, H.M., Park, M.V.D.Z., Gosens, I, De Jong, W. and Cassee, F.R. (2014): Physicochemical characteristics of nanomaterials that affect pulmonary inflammation. Part. Fibre Toxicol., 11, 18.

Castranova, V. and Mercer, R.R. (2012): Responses to pulmonary exposure to carbon nanotubes. In: Donaldson, K., Poland, C.A., Duffin, R., Bonner, J., (Eds.), The Toxicology of Carbon Nanotubes. Cambridge University Press, Cambridge, pp.134-149.

Costa, D.L., Lehmann, J.R., Winsett, D., Richards, J., Ledbetter, A.D. and Dreher, K.L. (2006): Comparative pulmonary toxicological assessment of oil combustion particles following inhala- 
Pulmonary toxicity induced by single-walled carbon nanotubes

tion or instillation exposure. Toxicol. Sci., 91, 237-246.

Donaldson, K., Aiken, R., Tran, L., Stone, V., Duffin, R., Forrest, G. and Alexander, A. (2006): Carbon nanotubes: a review of their properties in relation to pulmonary toxicology and workplace safety. Toxicol. Sci., 92, 5-22.

Donaldson, K., Murphy, F.A., Duffin, R. and Poland, C.A. (2010): Asbestos, carbon nanotubes and the pleural mesothelium: a review of the hypothesis regarding the role of long fibre retention in the parietal pleura, inflammation and mesothelioma. Part. Fibre Toxicol., 7, 5.

Driscoll, K.E., Costa, D.L., Hatch, G., Henderson, R., Oberdorster, G., Salem, H. and Schlesinger, R.B. (2000): Intratracheal instillation as an exposure technique for the evaluation of respiratory tract toxicity: uses and limitations. Toxicol. Sci., 55, 24-35.

Ema, M., Matsuda, A., Kobayashi, N., Naya, M. and Nakanishi, J. (2011): Evaluation of dermal and eye irritation and skin sensitization due to carbon nanotubes. Regul. Toxicol. Pharmacol., 61, 276-281.

Ema, M., Gamo, M. and Honda, K. (2016): A review of toxicity studies of single-walled carbon nanotubes in laboratory animals. Regul. Toxicol. Pharmacol., 74, 42-63.

Fujita, K., Fukuda, M., Endoh, S., Kato, H., Maru, J., Nakamura, A., Uchino, K., Shinohara, N., Obara, S., Nagano, R., Horie, M., Kinugasa, S., Hashimoto, H. and Kishimoto, A. (2013): Physical properties of single-wall carbon nanotubes in cell culture and their dispersal due to alveolar epithelial cell response. Toxicol. Mech. Methods, 23, 598-609.

Fujita, K., Fukuda, M., Endoh, S., Maru, J., Kato, H., Nakamura, A., Shinohara, N., Uchino, K. and Honda, K. (2015a): Size effects of single-walled carbon nanotubes on in vivo and in vitro pulmonary toxicity. Inhal. Toxicol., 27, 207-223.

Fujita, K., Fukuda, M., Fukui, H., Horie, M., Endoh, S., Uchida, K., Shichiri, M., Morimoto, Y., Ogami, A. and Iwahashi, H. (2015b): Intratracheal instillation of single-wall carbon nanotubes in the rat lung induces time-dependent changes in gene expression. Nanotoxicology, 9, 290-301.

Ge, C., Meng, L., Xu, L., Bai, R., Du, J., Zhang, L, Li, Y., Chang, Y., Zhao, Y. and Chen, C. (2012): Acute pulmonary and moderate cardiovascular responses of spontaneously hypertensive rats after exposure to single-wall carbon nanotubes. Nanotoxicology, 6, 526-542.

Hamilton, R.F., Wu, N., Porter, D., Buford, M., Wolfarth, M. and Holian, A. (2009): Particle length-dependent titanium dioxide nanomaterials toxicity and bioactivity. Part. Fibre Toxicol., 6, 35.

Hayter, A.J. (1984): A proof of the conjecture that the Tukey-Kramer multiple comparisons procedure is conservative. Ann. Statist., 12, 61-75.

Hubbard, R., Venn, A., Lewis, S. and Britton, J. (2000): Lung cancer and cryptogenic fibrosing alveolitis. A population-based cohort study. Am. J. Respir. Crit. Care Med., 161, 5-8.

Jiménez, A.S., Brouwer, D. and Van Tongeren, M. (2014): Workplace Inhalation Exposure to Engineered Nanomaterials. Detection, Measurement, and Assessment. In: Monteiro-Riviere, N.A., Tran, C.L., (Eds.), Nanotoxicology: Progress toward Nanomedicine, second ed., CRC Press, Boca Raton, pp.77-96.

Johnston, H.J., Hutchison, G.R., Christensen, F.M., Peters, S., Hankin, S., Aschberger, K. and Stone, V. (2010): A critical review of the biological mechanisms underlying the in vivo and in vitro toxicity of carbon nanotubes: The contribution of physico-chemical characteristics. Nanotoxicology, 4, 207-246.

Kramer, C.Y. (1956): Extension of multiple range tests to group means with unequal numbers of replications. Biometrics, 12, 307-310.

Lam, C.W., James, J.T., McCluskey, R. and Hunter, R.L. (2004): Pulmonary toxicity of single-wall carbon nanotubes in mice 7 and 90 days after intratracheal instillation. Toxicol. Sci., 77, 126134.

Landsiedel, R., Ma-Hock, L., Hofmann, T., Wiemann, M., Strauss, V., Treumann, S., Wohlleben, W., Gröters, S., Wiench, K. and van Ravenzwaay, B. (2014): Application of short-term inhalation studies to assess the inhalation toxicity of nanomaterials. Part. Fibre Toxicol., 11, 16.

Morimoto, Y., Hirohashi, M., Horie, M., Ogami, A., Oyabu, T., Myojo, T., Hashiba, M., Mizuguchi, Y., Kambara, T., Lee, B.W., Kuroda, E., Yamamoto, K., Kobayashi, N., Endoh, S., Uchida, K., Nakazato, T., Fujita, K., Nakanishi, J. and Tanaka, I. (2012): Pulmonary toxicity of well-dispersed single-wall carbon nanotubes following intratracheal instillation. J. Nano R., 18-19, 9-25.

Murray, A.R., Kisin, E., Leonard, S.S., Young, S.H., Kommineni, C., Kagan, V.E., Castranova, V. and Shvedova, A.A. (2009): Oxidative stress and inflammatory response in dermal toxicity of single-walled carbon nanotubes. Toxicology, 257, 161-171.

Murray, A.R., Kisin, E.R., Tkach, A.V., Yanamala, N., Mercer, R., Young, S.H., Fadeel, B., Kagan, V.E. and Shvedova, A.A. (2012): Factoring-in agglomeration of carbon nanotubes and nanofibers for better prediction of their toxicity versus asbestos. Part. Fibre Toxicol., 9, 10.

Naya, M., Kobayashi, N., Endoh, S., Maru, J., Honda, K., Ema, M., Tanaka, J., Fukumuro, M., Hasegawa, K., Nakajima, M., Hayashi, M. and Nakanishi, J. (2012): In vivo genotoxicity study of single-wall carbon nanotubes using comet assay following intratracheal instillation in rats. Regul. Toxicol. Pharmacol., 64, 124-129.

Poland, C.A., Duffin, R, Kinloch, I., Maynard, A., Wallace, W.A.H., Seaton, A., Stone, V., Brown, S., MacNee, W. and Donaldson, K. (2008): Carbon nanotubes introduced into the abdominal cavity of mice show asbestos-like pathogenicity in a pilot study. Nat. Nanotechnol., 3, 423-428.

Royen, T. (1989): Generalized maximum range tests for pairwise comparisons of several populations. Biom. J., 31, 905-929.

Schinwald, A., Murphy, F.A., Prina-Mello, A., Poland, C.A., Byrne, F., Movia, D., Glass, J.R., Dickerson, J.C., Schultz, D.A., Jeffree, C.E., MacNee, W. and Donaldson, K. (2012): The threshold length for fiber-induced acute pleural inflammation: shedding light on the early events in asbestos-induced mesothelioma. Toxicol. Sci., 128, 461-470.

Shaffer, J.P. (1986): Modified sequentially rejective multiple test procedures. J. Am. Stat. Assoc., 81, 826-831.

Shvedova, A.A., Kisin, E., Murray, A.R., Johnson, V.J., Gorelik, O., Arepalli, S., Hubbs, A.F., Mercer, R.R., Keohavong, P., Sussman, N., Jin, J., Yin, J., Stone, S., Chen, B.T., Deye, G., Maynard, A., Castranova, V., Baron, P.A. and Kagan, V.E. (2008): Inhalation vs. aspiration of single-walled carbon nanotubes in C57BL/6 mice: inflammation, fibrosis, oxidative stress, and mutagenesis. Am. J. Physiol. Lung Cell. Mol. Physiol., 295, L552-L565.

Shvedova, A.A., Kisin, E.R., Porter, D., Schulte, P., Kagan, V.E., Fadeel, B. and Castranova, V. (2009): Mechanisms of pulmonary toxicity and medical applications of carbon nanotubes: Two faces of Janus? Pharmacol. Ther., 121, 192-204.

Shvedova, A.A., Kisin, E.R., Murray, A.R., Mouithys-Mickalad, A., Stadler, K., Mason, R.P. and Kadiiska, M. (2014a): ESR evidence for in vivo formation of free radicals in tissue of mice 


\section{Ema et al.}

exposed to single-walled carbon nanotubes. Free Rad. Biol. Med., 73, 154-165.

Shvedova, A.A., Yanamala, N., Kisin, E.R., Tkach, A.V., Murray, A.R., Hubbs, A., Chirila, M.M., Keohavong, P., Sycheva, L.P., Kagan, V.E. and Castranova, V. (2014b): Long-term effects of carbon containing engineered nanomaterials and asbestos in the lung: one year postexposure comparisons. Am. J. Physiol. Lung Cell. Mol. Physiol., 306, L170-L182.

Shvedova, A., Pietroiusti, A. and Kagan, V. (2016): Nanotoxicology ten years later: lights and shadows. Toxicol. Appl. Pharmacol., 299, 1-2.

Singh, C. and Song, W. (2012): Carbon nanotube structure, synthesis, and applications. In: Donaldson, K., Poland, C.A., Duffin, R., Bonner, J., (Eds.), The Toxicology of Carbon Nanotubes. Cambridge University Press, Cambridge, pp.1-37.

Thomas, T.A. (2014): Nanotechnology in Consumer Products: addressing Potential Health and Safety Implications for Con- sumers. In: Monteiro-Riviere, N.A., Tran, C.L. (Eds.), Nanotoxicology: Progress toward Nanomedicine, second ed., CRC Press, Boca Raton, pp.97-112.

Tukey, J.W. (1953): The problem of multiple comparisons. Mimeographed monograph. Princeton Univ.

Warheit, D.B., Laurence, B.R., Reed, K.L., Roach, D.H., Reynolds, G.A. and Webb, T.R. (2004): Comparative pulmonary toxicity assessment of single-wall carbon nanotubes in rats. Toxicol. Sci., 77, 117-125.

WHO (World Health Organization) (2000): Concise International Chemical Assessment Document 24: Crystalline silica, quartz. Geneva.

Ye, J., Shi, X., Jones, W., Rojanasakul, Y., Cheng, N., SchweglerBerry, D., Baron, P., Deye, G.J., Li, C. and Castranova, V. (1999): Critical role of glass fiber length in TNF- $\alpha$ production and transcription factor activation in macrophages. Am. J. Physiol., 276, L426-L434. 